\title{
STATIONARY SETS AND ASYMPTOTIC BEHAVIOR OF THE MEAN CURVATURE FLOW WITH FORCING IN THE PLANE
}

\author{
NICOLA FUSCO, VESA JULIN, MASSIMILIANO MORINI
}

\begin{abstract}
We consider the flat flow solutions of the mean curvature equation with a forcing term in the plane. We prove that for every constant forcing term the stationary sets are given by a finite union of disks with equal radii and disjoint closures. On the other hand for every bounded forcing term tangent disks are never stationary. Finally in the case of an asymptotically constant forcing term we show that the only possible long time limit sets are given by disjoint unions of disks with equal radii and possibly tangent.
\end{abstract}

\section{INTRODUCTION}

Mean curvature flow is one of the simplest and yet most interesting geometric evolution equation. In order to deal with formation of singularities or rough initial data several notions of generalized solutions have been proposed. Among them we mention Brakke's solutions in the varifold sense [7], level-set solutions in the viscosity sense [10], [15], De Giorgi's minimal barriers [12] and the flat flows solutions constructed by the minimizing movements method [2], [21]. Each method has its own advantages and drawbacks. For instance Brakke's theory fails to provide unique solutions, but yields a satisfactory partial regularity theory, see also [19]. On the contrary, the viscosity level-set method provides uniqueness and global existence, but it is not so convenient as far as regularity is concerned. Indeed in this framework one may construct singular solutions where the evolving hypersurfaces become sets with nonempty interior, the so called fattening phenomenon. This phenomenon can occur even if the initial set is regular after a positive time, see [5]. De Giorgi's minimal barriers provide essentially the same solutions as the level-set method, see [4]; within this approach the fattening phenomenon is related to the fact that minimal and maximal solutions may be different, see [5]. Flat flow solutions are also defined globally in time. They are always given by evolving boundaries of sets and may not be unique whenever the level-set solution experiences the fattening phenomenon. However, level-set solutions, De Giorgi's minimal barriers and flat flows all coincide with the classical solutions as long as the latter exist.

In this paper we focus on the flat flow approach for the mean curvature equation with a time dependent forcing term in the plane, i.e.,

$$
V_{t}=-k_{E_{t}}+f(t) \quad \text { on } \partial E_{t}
$$

with an arbitrary initial datum under the assumption that the forcing term $f$ is uniformly bounded, i.e.,

$$
\sup _{t \geq 0}|f(t)| \leq C_{0}
$$

Here $k_{E_{t}}$ stands for the curvature of the boundary of $E_{t}$ with respect to the orientation given by the outward normal. For the precise definition of flat flow see the beginning of Section 2 .

Key words and phrases. Forced mean curvature flow, large time behavior, stationary sets, critical sets. 
The existence of flat flow solutions for the equation (1.1) in any dimension and their relations with the De Giorgi' barriers and the level-set solutions has been investigated in [9]. In this paper we further elaborate on the properties of flat flows solutions in two dimensions focusing on the following issues: how the flat flow selects a solution when the fattening phenomenon occurs, the characterization of sets that are stationary when $f$ is constant and the long time behavior of solutions.

1.1. Flat flow as a selection principle. Here we consider a particular situation where the initial set is given by two tangent disks of equal radii $D_{r}\left(x_{1}\right)$ and $D_{r}\left(x_{2}\right)$. It is well known that in this example the level-set solution develops instantaneously a nonempty interior. When $f(t) \equiv 1 / r$ the minimal barrier solution of (1.1) is stationary, while the maximal barrier solution becomes a connected set containing a ball centered at the origin with a time dependent radius, see [5]. It is an interesting problem to look for a selection principle among the possible admissible behaviors. One such principle can be obtained by adding to the forcing term a small stochastic perturbation. This has been investigated in [14] where the perturbation considered is of the form $\varepsilon d W$, with $W$ a standard Brownian motion. The authors show that when $\varepsilon$ goes to zero the corresponding motion converges with probability $1 / 2$ to the maximal barrier solution and with probability $1 / 2$ to the minimal one. In this paper we prove that any flat flow instantaneously connects the two tangent disks with a thin neck and keeps enlarging the neck at least for a short time interval, thus showing that the flat flow somehow picks the behavior of the maximal barrier solution. The precise statement is as follows.

Theorem 1.1. Let $E_{0} \subset \mathbb{R}^{2}$ be a union of two tangent disks $E_{0}=D_{r}\left(x_{1}\right) \cup D_{r}\left(x_{2}\right)$ and let $\left(E_{t}\right)_{t}$ be a flat flow of (1.1) starting from $E_{0}$ and assume that (1.2) holds. There exist $\delta>0$, $\eta>0$ and $c>0$ such that for every $t \in(0, \delta)$ the set $E_{t}$ contains a dumbbell shaped simply connected set which in turn contains the disks $D_{\eta r}\left(x_{1}\right)$ and $D_{\eta r}\left(x_{2}\right)$ and a ball centered at the origin of radius $t$. In particular for every $t \in(0, \delta)$

$$
\left|E_{t} \backslash E_{0}\right| \geq c t^{3} \text {. }
$$

This theorem is also relevant for the second issue we want to deal with, i.e., the characterization of stationary sets, as it shows that the union of two equal tangent disks is not stationary for the flat flow.

1.2. Characterization of stationary sets. When the forcing term $f \equiv c_{0}$ equation (1.1) can be regarded as the gradient flow of the following energy

$$
\mathcal{E}(E)=P(E)-c_{0}|E|,
$$

where $P(E)$ stands for the perimeter of $E$ and $|E|$ for its Lebesgue measure. Therefore one might think that $E_{0}$ is stationary for the flow if and only if it is critical for the energy (1.3), i.e., it satisfies $k_{E_{0}}=c_{0}$ on $\partial E_{0}$ in a weak sense. Indeed if $E_{0}$ is stationary then it also critical, while the converse is certainly true when $E_{0}$ is smooth, i.e., is given by a union of finitely many disks with equal radii and mutually disjoint closures (see [13] for a characterization of critical sets in any dimension, even in the nonsmooth case). However, Theorem 1.1 shows that the two notions do not coincide since the union of two tangent disks of equal radii is critical as it has constant mean curvature in the weak sense, but not stationary. Here we show that a set $E$ is stationary for the flow (1.1) when $f \equiv c_{0}$ if and only if it is a union of disks with radius $r=1 / c_{0}$ with positive distance to each other. More precisely we have the following. 
Theorem 1.2. Assume $E_{0} \subset \mathbb{R}^{2}$ is a bounded set of finite perimeter. Then $E_{0}$ is stationary (see Definition 3.1) for the flow (1.1) with $f \equiv c_{0}$ if and only if there are points $x_{1}, \ldots, x_{N}$ such that $\left|x_{i}-x_{j}\right|>2 r$ for $i \neq j$, with $r=1 / c_{0}$, and

$$
E_{0}=\bigcup_{i=1}^{N} D_{r}\left(x_{i}\right) .
$$

The fact that any stationary set is a union of disjoint disks follows from a sharp quantitative version of the Alexandrov theorem in the plane, see Lemma 3.2, while the fact that the disks must be at positive distance apart is a consequence of Theorem 1.1.

We remark that the same type of classification holds true in the framework of level-set solutions, as recently shown in [16, Theorem 4.7]. The general $n$-dimensional case remains open also for the viscosity solutions, see [17].

1.3. Long time behavior. We now address the long time behavior of the flat flow under the assumption that the forcing term is asymptotically constant, namely that it satisfies

$$
\int_{0}^{\infty}\left|f(s)-c_{0}\right|^{2} d s<\infty
$$

In the next theorem our goal is to characterize the possible limit sets and we show in particular that the asymptotically stationary sets are given once again by a union of disjoint disks, which however can be tangent. Precisely we show that either, up to a diverging sequence $t_{j}$ of times, the area of $E_{t_{j}}$ blows up or the sets $\left(E_{t}\right)_{t}$ converge up to a translation in the Hausdorff sense to a disjoint union of disks with equal radii.

Theorem 1.3. Assume $E_{0} \subset \mathbb{R}^{2}$ is a bounded set of finite perimeter. Let $\left(E_{t}\right)_{t}$ be a flat flow of (1.1) starting from $E_{0}$ and assume (1.2) and (1.4) with $c_{0}>0$, and

$$
\sup _{t>0}\left|E_{t}\right|<\infty .
$$

Then there exist $N \in \mathbb{N}$ and $x_{i}(t):(0,+\infty) \rightarrow \mathbb{R}^{2}$, with $i=1, \ldots, N$ and $\left|x_{i}(t)-x_{j}(t)\right| \geq 2 / c_{0}$ for $i \neq j$, such that, setting $F_{t}=\cup_{i=i}^{N} D_{1 / c_{0}}\left(x_{i}(t)\right)$

$$
\lim _{t \rightarrow \infty} \sup _{x \in E_{t} \Delta F_{t}} d_{\partial F_{t}}(x)=0 .
$$

We stress here the fact that the initial set $E_{0}$ in the above theorem is an arbitrary bounded set of finite perimeter without further regularity assumption. It is plausible that in Theorem 1.3 the convergence holds not just up to translation.

Previous results dealt with special classes of sets in any dimension such as convex or starshaped initial sets, see for instance [3] and [20]. We also mention [23] where the long-time behavior of the discrete Euler implicit scheme for the volume preserving mean curvature flow is addressed for any arbitrary bounded initial set with finite perimeter. The long time behavior of the forced mean curvature flow in the context of viscosity level-set solutions was also investigated in [17] and [16] where it is shown that under certain assumptions the solutions converge to a stationary solution of the level-set equation. The problem of classifying the latter is open in general.

We now show that it is indeed possible to obtain as a limit of the flow (1.1) a union of essentially disjoint disks such that at least two of them are tangent. To this end we take $G$ to be the ellipse

$$
G=\left\{\left(x_{1}, x_{2}\right) \in \mathbb{R}^{2}: a^{2} x_{1}^{2}+x_{2}^{2}<1\right\} \quad \text { with } a>1
$$




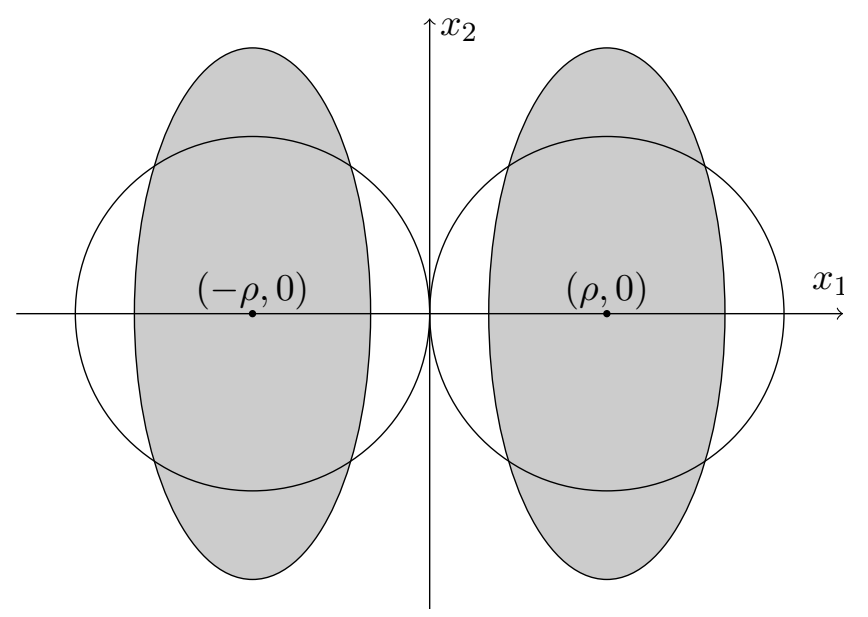

Figure 1.1. The union of two ellipses converges to the union of two tangent disks.

and we show the following theorem.

Theorem 1.4. Let $e_{1}=(1,0)$ and $G$ as above. Denote by $\rho=\frac{1}{\sqrt{a}}$ the radius such that $\left|D_{\rho}\right|=|G|$. The volume preserving mean curvature flow $\left(E_{t}\right)_{t}$, starting from

$$
E_{0}=\left(G-\rho e_{1}\right) \cup\left(G+\rho e_{1}\right),
$$

is well defined in the classical sense for all $t>0$ and converges exponentially fast to the union of two tangent disks

$$
E_{t} \rightarrow\left(D_{\rho}-\rho e_{1}\right) \cup\left(D_{\rho}+\rho e_{1}\right) .
$$

Note that Theorem 1.4 shows that a flat flow of (1.1) may converge to tangent disks. Indeed the classical solution of the flow in Theorem 1.4 is well defined and smooth for all times and we may write it in the form (1.1) with $f(t)=f_{\partial E_{t}} k_{E_{t}}$ and the flat flow agrees with it. Moreover, by the exponential convergence we have that $f(t)$ satisfies (1.4).

We note that in Theorem 1.4 the flow $\left(E_{t}\right)_{t}$ remains smooth and diffeomorphic to a union of two disks. Only the limit set is non-smooth.

\section{Notation and Preliminary Results}

Since the results of this section hold in any dimension we state them in full generality and we will go back to the planar case in the next sections.

Given a set $A \subset \mathbb{R}^{n}$ the distance function $d_{A}: \mathbb{R}^{n} \rightarrow[0, \infty)$ is defined as usual

$$
d_{A}(x):=\inf _{y \in A}|x-y|
$$

and we denote the signed distance function by $\bar{d}_{A}: \mathbb{R}^{n} \rightarrow \mathbb{R}$,

$$
\bar{d}_{A}(x):=\left\{\begin{array}{l}
-d_{\mathbb{R}^{n} \backslash A}(x), \text { for } x \in A \\
d_{A}(x), \text { for } x \in \mathbb{R}^{n} \backslash A .
\end{array}\right.
$$

Then clearly it holds $d_{\partial A}=\left|\bar{d}_{A}\right|$.

For a set of finite perimeter $E \subset \mathbb{R}^{n}$ we denote its perimeter by $P(E)$ and recall that for regular enough set it holds $P(E)=\mathcal{H}^{n-1}(\partial E)[1,22]$. For a measurable set $|E|$ denotes its Lebesgue measure. We denote by $H_{E}$ the sum of the principal curvatures of $E$, while in the 
planar case we write $k_{E}$. We denote the disk with radius $r$ centered at $x$ by $D_{r}(x)$ and in the higher dimensional case we write $B_{r}(x)$ instead.

We consider solutions of (1.1) constructed via the minimizing movement scheme. We fix a small time step $h>0$ and a bounded set of finite perimeter $E_{0} \subset \mathbb{R}^{n}$, which is our initial set $E^{h, 0}=E_{0}$. We obtain a sequence of set $\left(E^{h, k}\right)_{k=1}^{\infty}$ by iterative minimizing procedure, where $E^{h, k+1}$ is a minimizer of the functional $\mathcal{F}_{k}\left(E ; E^{h, k}\right)$ defined as

$$
\mathcal{F}_{k}\left(E ; E^{h, k}\right)=P(E)+\frac{1}{h} \int_{E} \bar{d}_{E^{h, k}} d x-\bar{f}(k h)|E|,
$$

where $\bar{d}_{E^{h, k}}$ is the signed distance defined above and $\bar{f}(k h)=f_{k h}^{(k+1) h} f(s) d s$. We define the approximate flat flow $\left(E_{t}^{h}\right)_{t>0}$ by

$$
E_{t}^{h}=E^{h, k}, \quad \text { for }(k-1) h<t \leq k h
$$

and we set $\bar{f}(t)=\bar{f}(k h)$ for $(k-1) h<t \leq k h$. Any cluster point of $E_{t}^{h}$ as $h$ goes to zero is called a flat flow for the equation (1.1).

We warn the reader that in the above definition it is understood that we identify $E^{h, k}$ with its set of its points of density 1 so that there is no ambiguity in the definition of $\bar{d}_{E^{h, k}}$.

Recall that if $E_{0}$ and $f$ are smooth then any flat flow coincide with the classical solution of (1.1) as long as the latter remains smooth, see [9].

In general, the problem (2.1) does not admit a unique minimizer and thus there is no unique way to define the approximate flat flow $\left(E_{t}^{h}\right)_{t>0}$. Also the flat flow may not be unique when fattening occurs. However, as we mentioned in the introduction, in the case when the initial set and the forcing term are smooth, the flat flow is unique for a short time interval and agrees with the classical solution.

Even if there is no uniqueness, the approximate flat flow satisfies the following weak comparison principle, see for instance the proof of Lemma 6.2 in [8].

Proposition 2.1. Assume $f_{1}, f_{2}:[0, \infty) \rightarrow \mathbb{R}$ satisfy (1.2). Let $E_{0}, F_{0}$ be two bounded sets of finite perimeter and let $\left(E_{t}^{h}\right)_{t}$ be an approximate flat flow with forcing term $f_{1}$ starting from $E_{0}$ and $\left(F_{t}^{h}\right)_{t}$ an approximate flat flow with forcing term $f_{2}$ starting from $F_{0}$.

(i) If $F_{0} \subset E_{0}$ and $f_{1}>f_{2}$, then for every $t>0$ it holds $F_{t}^{h} \subset E_{t}^{h}$.

(ii) If $E_{0} \subset \mathbb{R}^{n} \backslash F_{0}$ and $-f_{2}>f_{1}$, then for every $t>0$ it holds $E_{t}^{h} \subset \mathbb{R}^{n} \backslash F_{t}^{h}$.

We need preliminary results on the structure of the approximate flat flow constructed via (2.1). We note that if $E^{h, k+1}$ is a minimizer of $\mathcal{F}_{k}\left(\cdot, E^{h, k}\right)$ then it is a $\Lambda$-minimizer of the perimeter, see for instance [24], with $\Lambda \leq C / h$, see [22] for the definition of $\Lambda$-minimizer. Then it follows that $\partial E^{h, k+1}$ is $C^{1, \alpha}$-regular for all $\alpha \in(0,1)$ up to a singular set $\Sigma$ with Hausdorff dimension at most $n-8$, see [22]. Then the Euler-Lagrange equation

$$
\frac{\bar{d}_{E^{h, k}}}{h}=-H_{E^{h, k+1}}+\bar{f}(k h) \quad \text { on } \partial E^{h, k+1} \backslash \Sigma,
$$

which holds in the weak sense, implies that $\partial E^{h, k+1} \backslash \Sigma$ is $C^{2, \alpha}$-regular and satisfies (2.3) in the classical sense.

Lemma 2.2. Assume that $\left(E^{h, k}\right)_{k}$ is a sequence obtained via minimizing movements (2.1) starting from a bounded set of finite perimeter $E_{0}$ and assume that the forcing term satisfies (1.2). Then there is a constant $C_{1}$ such that for every $k=0,1,2, \ldots$

$$
\sup _{x \in E^{h, k+1} \Delta E^{h, k}} d_{\partial E^{h, k}}(x) \leq C_{1} \sqrt{h} .
$$


Moreover, there are constants $C_{2}>1$ and $c_{1}>0$ such that for every $k=1,2,3, \ldots$ it holds

$$
\left|E^{h, k+1} \Delta E^{h, k}\right| \leq C_{2}\left(l P\left(E^{h, k}\right)+\frac{1}{l} \int_{E^{h, k+1} \Delta E^{h, k}}\left|\bar{d}_{E^{h, k}}(x)\right| d x\right)
$$

for any $0<l<c_{1} \sqrt{h}$.

Proof. The first claim follows from the argument of the proof of [24, Proposition 3.2] and thus we omit it. The second claim follows from an argument similar to [24, Proposition 3.4] and we only sketch it. We write

$\left|E^{h, k+1} \Delta E^{h, k}\right|=\left|\left\{x \in E^{h, k+1} \Delta E^{h, k}:\left|\bar{d}_{E^{h, k}}(x)\right| \geq l\right\}\right|+\left|\left\{x \in E^{h, k+1} \Delta E^{h, k}:\left|\bar{d}_{E^{h, k}}(x)\right|<l\right\}\right|$.

We estimate the first term as

$$
\left|\left\{x \in E^{h, k+1} \Delta E^{h, k}:\left|\bar{d}_{E^{h, k}}(x)\right| \geq l\right\}\right| \leq \frac{1}{l} \int_{E^{h, k+1} \Delta E^{h, k}}\left|\bar{d}_{E^{h, k}}(x)\right| d x .
$$

For the second term we use Vitali covering theorem to choose a finite family of disjoint balls $\left(B_{l}\left(x_{i}\right)\right)_{i=1}^{N}$, with $x_{i} \in \partial E^{h, k}$, such that

$$
\left\{x \in \mathbb{R}^{n}:\left|\bar{d}_{E^{h, k}}(x)\right|<l\right\} \subset \cup_{i=1}^{N} B_{5 l}\left(x_{i}\right) .
$$

Since $E^{h, k}$ is a minimizer of $\mathcal{F}_{k}\left(E ; E^{h, k-1}\right)$, we have the density estimates [24, Corollary 3.3]. Thus by the relative isoperimetric inequality we have for every $i=1, \ldots, N$

$$
\left|B_{l}\left(x_{i}\right)\right| \leq C\left(\mathcal{H}^{n-1}\left(\partial E^{h, k} \cap B_{l}\left(x_{i}\right)\right)^{\frac{n}{n-1}} \leq C l \mathcal{H}^{n-1}\left(\partial E^{h, k} \cap B_{l}\left(x_{i}\right)\right) .\right.
$$

Therefore

$$
\begin{aligned}
\left|\left\{x \in E^{h, k+1} \Delta E^{h, k}:\left|\bar{d}_{E^{h, k}}(x)\right|<l\right\}\right| & \leq \sum_{i=1}^{N}\left|B_{5 l}\left(x_{i}\right)\right| \leq 5^{n} \sum_{i=1}^{N}\left|B_{l}\left(x_{i}\right)\right| \\
& \leq C l \sum_{i=1}^{N} \mathcal{H}^{n-1}\left(\partial E^{h, k} \cap B_{l}\left(x_{i}\right)\right) \leq C l P\left(E^{h, k}\right) .
\end{aligned}
$$

In the next proposition we list useful properties of the flow in the case when the forcing term satisfies only (1.2).

Proposition 2.3. Let $\left(E_{t}^{h}\right)_{t}$ be an approximate flat flow starting from a bounded set of finite perimeter $E_{0}$ and assume that the forcing term satisfies (1.2). Then the following hold:

(i) For every $T>0$ there is $R_{T}>0$ such that $E_{t}^{h} \subset B_{R_{T}}$ for every $t \leq T$.

(ii) There is $C_{3}$, depending only on $E_{0}$ and $f$, such that for every $T>0$ it holds

$$
P\left(E_{T}^{h}\right) \leq C_{3}^{1+T}
$$

for $h$ sufficiently small.

(iii) For every $h<s<t<T$ with $t-s>h$ and $h$ sufficiently small, it holds $\left|E_{t}^{h} \Delta E_{s}^{h}\right| \leq$ $C_{T} \sqrt{t-s}$, where the constant $C_{T}$ depends on $T$.

(iv) There exists a subsequence $\left(h_{l}\right)_{l}$ converging to zero such that $\left(E_{t}^{h_{l}}\right)_{t}$ converges to a flat flow $\left(E_{t}\right)_{t}$ in $L^{1}$ in space and locally uniformly in time, i.e., for every $T$

$$
\sup _{h_{l}<t \leq T}\left|E_{t}^{h_{l}} \Delta E_{t}\right| \rightarrow 0 \quad \text { as } h_{l} \rightarrow 0 .
$$


Proof. The claim (i) follows by applying Proposition 2.1 to $E_{t}^{h}$ and $F_{t}^{h}$, where the latter is approximate flat flow starting from $B_{R}$, such that $E_{0} \subset B_{R}$, and with constant forcing term $f_{2} \equiv \sup _{t} f(t)+1$. Then $E_{t}^{h} \subset F_{t}^{h}$. It is easy to check that the sets $\left(F_{t}^{h}\right)_{t \leq T}$ are balls whose radii satisfy $r(t) \leq C(1+T)$ for $t \leq T$.

Let us prove (ii). By the minimality of $E^{h, k+1}$ we have $\mathcal{F}_{k}\left(E^{h, k+1} ; E^{h, k}\right) \leq \mathcal{F}_{k}\left(E^{h, k} ; E^{h, k}\right)$ which implies

$$
P\left(E^{h, k+1}\right)+\frac{1}{h} \int_{E^{h, k+1}} \bar{d}_{E^{h, k}} d x-\bar{f}(k h)\left|E^{h, k+1}\right| \leq P\left(E^{h, k}\right)+\frac{1}{h} \int_{E^{h, k}} \bar{d}_{E^{h, k}} d x-\bar{f}(k h)\left|E^{h, k}\right| .
$$

We write this as

$$
\frac{1}{h} \int_{E^{h, k+1} \Delta E^{h, k}}\left|\bar{d}_{E^{h, k}}\right| d x+P\left(E^{h, k+1}\right) \leq P\left(E^{h, k}\right)+\bar{f}(k h)\left(\left|E^{h, k+1}\right|-\left|E^{h, k}\right|\right) .
$$

By (1.2) we simply estimate $\bar{f}(k h)\left(\left|E^{h, k+1}\right|-\left|E^{h, k}\right|\right) \leq C_{0}\left|E^{h, k+1} \Delta E^{h, k}\right|$. Then we use the second statement in Lemma 2.2 with $l=\hat{C} h$, where $\hat{C}$ is a large constant to deduce

$$
\left|E^{h, k+1} \Delta E^{h, k}\right| \leq C h P\left(E^{h, k}\right)+\frac{1}{2 C_{0} h} \int_{E^{h, k+1} \Delta E^{h, k}}\left|\bar{d}_{E^{h, k}}\right| d x .
$$

Therefore we deduce from these two inequalities and from (2.4) that

$$
\frac{1}{2 h} \int_{E^{h, k+1} \Delta E^{h, k}}\left|\bar{d}_{E^{h, k}}\right| d x+P\left(E^{h, k+1}\right) \leq(1+C h) P\left(E^{h, k}\right) .
$$

By iterating the inequality $P\left(E^{h, k+1}\right) \leq(1+C h) P\left(E^{h, k}\right)$ we get

$$
P\left(E^{h, k}\right) \leq(1+C h)^{k-1} P\left(E^{h, 1}\right)=\left((1+C h)^{1 / h}\right)^{(k-1) h} P\left(E^{h, 1}\right) \leq C^{(k-1) h} P\left(E^{h, 1}\right) .
$$

Finally we use (2.4) for $k=0$ and have

$$
P\left(E^{h, 1}\right) \leq P\left(E_{0}\right)+\bar{f}(h)\left(\left|E^{h, 1}\right|-\left|E_{0}\right|\right) .
$$

By (i) we may estimate $\left|E^{h, 1}\right| \leq\left|B_{2 R}\right|$ for $h$ sufficiently small, where we recall that $B_{R}$ is the ball containing $E_{0}$. Therefore $P\left(E^{h, 1}\right) \leq P\left(E_{0}\right)+C$ and we obtain the claim (ii)

The claim (iii) follows from argument similar to [24, Proposition 3.5] so we only point out the main differences. Let $k, m$ be such that $s \in(k h,(k+1) h]$ and $t \in((k+m) h,(k+m+1) h]$. Note that $m h \leq 2(t-s)$. We may estimate the quantity $\left|E_{t}^{h} \Delta E_{s}^{h}\right|$ by applying the second statement of Lemma 2.2 with $l=c_{1} \frac{h}{2 \sqrt{t-s}},(2.5)$ and the part (ii) to get

$$
\begin{aligned}
\left|E_{t}^{h} \Delta E_{s}^{h}\right| & \leq \sum_{i=1}^{m}\left|E^{h, k+i+1} \Delta E^{h, k+i}\right| \\
& \leq \sum_{i=1}^{m} C\left(\frac{h}{\sqrt{t-s}} P\left(E^{h, k+i}\right)+\frac{\sqrt{t-s}}{h} \int_{E^{h, k+i+1} \Delta E^{h, k+i}}\left|\bar{d}_{E^{h, k+i}}(x)\right| d x\right) \\
& \leq \sum_{i=1}^{m} C\left(\frac{h}{\sqrt{t-s}} P\left(E^{h, k+i}\right)+\sqrt{t-s}\left((1+C h) P\left(E^{h, k+i}\right)-P\left(E^{h, k+i+1}\right)\right)\right) \\
& \leq C \sqrt{t-s} \sup _{t \leq T} P\left(E_{t}^{h}\right)+\sqrt{t-s} P\left(E^{h, k+1}\right) \leq C_{T} \sqrt{t-s} .
\end{aligned}
$$

Similarly (iv) follows from the proof of [24, Theorem 2.2]. 
When in addition we assume that the forcing term satisfies (1.4) we obtain estimates which are more uniform with respect to time. To this aim we define the following quantity which plays the role of the energy

$$
\mathcal{E}(E):=P(E)-c_{0}|E|,
$$

where $c_{0}$ is the constant appearing in (1.4).

Proposition 2.4. Let $\left(E_{t}^{h}\right)_{t}$ be an approximate flat flow starting from a bounded set of finite perimeter $E_{0}$ and assume that the forcing term satisfies (1.2) and (1.4). Then, if $h$ is sufficiently small, the following hold:

(i) For every $\varepsilon>0$ there is $T_{\varepsilon}$ such that for every $T_{\varepsilon}<T_{1}<T_{2}$, with $T_{2} \geq T_{1}+h$, we have the following dissipation inequality

$$
c \int_{T_{1}}^{T_{2}} \int_{\partial E_{t}^{h}}\left(H_{E_{t}^{h}}-\bar{f}(t-h)\right)^{2} d \mathcal{H}^{n-1} d t+\mathcal{E}\left(E_{T_{2}}^{h}\right) \leq \mathcal{E}\left(E_{T_{1}-h}^{h}\right)+\varepsilon \sup _{T_{1}-h \leq t \leq T_{2}} P\left(E_{t}^{h}\right) .
$$

(ii) If $\sup _{t \geq 0}\left|E_{t}^{h}\right|<\infty$, then $\sup _{t \geq 0} P\left(E_{t}^{h}\right)<\infty$.

(iii) If $\sup _{t \geq 0}\left|E_{t}^{h}\right|<\infty$, there exists a constant $C_{4}$ such that $\left|E_{t}^{h} \Delta E_{s}^{h}\right| \leq C_{4} \sqrt{t-s}$ for every $h<s<t$ with $t-s>h$.

Proof. To prove (i) we begin with (2.4). This time we estimate the last term in (2.4) as

$$
\bar{f}(k h)\left(\left|E^{h, k+1}\right|-\left|E^{h, k}\right|\right) \leq c_{0}\left(\left|E^{h, k+1}\right|-\left|E^{h, k}\right|\right)+\left|\bar{f}(k h)-c_{0}\right|\left|E^{h, k+1} \Delta E^{h, k}\right| .
$$

We use the second estimate in Lemma 2.2 with $l=\hat{C}\left|\bar{f}(k h)-c_{0}\right| h$, where $\hat{C}$ is a large constant and $h$ is sufficiently small, to deduce

$$
\left|\bar{f}(k h)-c_{0}\right|\left|E^{h, k+1} \Delta E^{h, k}\right| \leq C\left|\bar{f}(k h)-c_{0}\right|^{2} h P\left(E^{h, k}\right)+\frac{1}{2 h} \int_{E^{h, k+1} \Delta E^{h, k}}\left|\bar{d}_{E^{h, k}}\right| d x .
$$

Therefore we have by (2.4) that

$$
\frac{1}{2 h} \int_{E^{h, k+1} \Delta E^{h, k}}\left|\bar{d}_{E^{h, k}}\right| d x+\mathcal{E}\left(E^{h, k+1}\right) \leq \mathcal{E}\left(E^{h, k}\right)+C\left|\bar{f}(k h)-c_{0}\right|^{2} h P\left(E^{h, k}\right),
$$

where $\mathcal{E}$ is defined in (2.7).

Let us fix $\varepsilon>0$. Since we assume (1.4), there exists $T_{\varepsilon}$ such that

$$
\int_{T_{\varepsilon}}^{\infty}\left(f(t)-c_{0}\right)^{2} d t \leq \frac{\varepsilon}{C}
$$

where $C$ is a constant to be chosen later. Let $T_{2}>T_{1}>T_{\varepsilon}$ and let $j, m$ be such that $T_{1} \in(j h,(j+1) h]$ and $T_{2} \in((j+m) h,(j+m+1) h]$. We iterate the previous inequality from 
$k=j-1$ to $k=j+m-1$ and obtain

$$
\begin{aligned}
\sum_{k=j}^{j+m} \frac{1}{2 h} \int_{E^{h, k+1} \Delta E^{h, k}} & \left|\bar{d}_{E^{h, k}}\right| d x+\mathcal{E}\left(E_{T_{2}}^{h}\right) \\
& \leq \mathcal{E}\left(E_{T_{1}-h}^{h}\right)+C\left(\sup _{T_{1}-h \leq t \leq T_{2}} P\left(E_{t}^{h}\right)\right)\left(\int_{T_{1}-h}^{T_{2}}\left|\bar{f}(t)-c_{0}\right|^{2} d t\right) \\
& \leq \mathcal{E}\left(E_{T_{1}-h}^{h}\right)+C\left(\sup _{T_{1}-h \leq t \leq T_{2}} P\left(E_{t}^{h}\right)\right)\left(\int_{T_{\varepsilon}}^{\infty}\left|f(t)-c_{0}\right|^{2} d t\right) \\
& \leq \mathcal{E}\left(E_{T_{1}-h}^{h}\right)+\varepsilon\left(\sup _{T_{1}-h \leq t \leq T_{2}} P\left(E_{t}^{h}\right)\right)
\end{aligned}
$$

where the last inequality follows from (2.8).

Arguing as in the proof of [24, Lemma 3.6], we deduce that there is a constant $c>0$, depending only on the dimension, such that

$$
c h \int_{\partial E^{h, k+1}}\left(\frac{\bar{d}_{E^{h, k}}}{h}\right)^{2} d \mathcal{H}^{n-1} \leq \int_{E^{h, k+1} \Delta E^{h, k}} \frac{\left|\bar{d}_{E^{h, k}}\right|}{h} d x .
$$

Therefore by the Euler-Lagrange equation (2.3) we have

$$
\begin{aligned}
\sum_{k=j}^{j+m} \frac{1}{h} \int_{E^{h, k+1} \Delta E^{h, k}}\left|\bar{d}_{E^{h, k}}\right| d x & \geq c \sum_{k=j}^{j+m} h \int_{\partial E^{h, k+1}}\left(\frac{\bar{d}_{E^{h, k}}}{h}\right)^{2} d \mathcal{H}^{n-1} \\
& =c \sum_{k=j}^{j+m} h \int_{\partial E^{h, k+1}}\left(H_{E^{h, k+1}}-\bar{f}(k h)\right)^{2} d \mathcal{H}^{n-1} \\
& \geq c \int_{T_{1}}^{T_{2}} \int_{\partial E_{t}^{h}}\left(H_{E_{t}^{h}}-\bar{f}(t-h)\right)^{2} d \mathcal{H}^{n-1} d t
\end{aligned}
$$

Thus we have the claim (i) by (2.9).

To show (ii) we fix $0<\varepsilon<1 / 2, T>T_{\varepsilon}$ and apply the part (i) with $T_{1}=T_{\varepsilon}+h$ and $T_{1}+h<T_{2}=t \leq T$ to deduce

$$
\mathcal{E}\left(E_{t}^{h}\right) \leq \mathcal{E}\left(E_{T_{\varepsilon}}^{h}\right)+\varepsilon \sup _{T_{\varepsilon} \leq s \leq T} P\left(E_{s}^{h}\right) .
$$

We recall that $\mathcal{E}(E)=P(E)-c_{0}|E|$ and that we assume $\sup _{t>0}\left|E_{t}^{h}\right|<\infty$. Therefore from the above inequality, recalling that $P\left(E_{t}^{h}\right) \leq C_{\varepsilon}$ for all $t<T_{\varepsilon}+1$ by Proposition 2.3 (ii), we get

$$
P\left(E_{t}^{h}\right) \leq C_{\varepsilon}+c_{0} \sup _{t>0}\left|E_{t}^{h}\right|+\varepsilon \sup _{T_{\varepsilon} \leq s \leq T} P\left(E_{s}^{h}\right)
$$

for every $T_{\varepsilon}<t \leq T$. Thus, since $\varepsilon<1 / 2$ we deduce that

$$
\sup _{T_{\varepsilon} \leq t \leq T} P\left(E_{t}^{h}\right) \leq 2\left(C_{\varepsilon}+c_{0} \sup _{t>0}\left|E_{t}^{h}\right|\right) .
$$

The claim (ii) follows from the fact that $T$ was arbitrary.

Finally the proof of (iii) follows from the proof of Proposition 2.3 (iii), noticing that now the constant $C_{T}$ is in fact independent on $T$ thanks to the bound on the perimeters provided by (ii). 
Remark 2.5. If $\left(E_{t}^{h}\right)_{t}, E_{0}$ and $f$ are as in Proposition 2.4, and if we assume

$$
\sup _{t \geq 0}\left|E_{t}^{h}\right| \leq C,
$$

then Proposition (i) and (ii) imply that the energy $\mathcal{E}\left(E_{t}^{h}\right)$ is asymptotically almost decreasing. More precisely, for every $\varepsilon>0$ there is $T_{\varepsilon}$ such that for $t>s>T_{\varepsilon}$ it holds

$$
\mathcal{E}\left(E_{t}^{h}\right) \leq \mathcal{E}\left(E_{s}^{h}\right)+C \varepsilon,
$$

with $T_{\varepsilon}$ and $C$ independent of $h$. This inequality implies in particular that there exists

$$
\lim _{t \rightarrow+\infty} \mathcal{E}\left(E_{t}^{h}\right)
$$

Moreover, from the proof of Proposition 2.4 we have also that if $h$ is sufficiently small and $\sup _{0<t<T}\left|E_{t}^{h}\right| \leq C$ for some $T>0$, then there exists a constant $\tilde{C}$, independent of $h$, such that $\sup _{0<t<T} P\left(E_{t}^{h}\right) \leq \tilde{C}$.

\section{Stationary SETS AND PROOF OF TheOREM 1.1}

In this section we go back to the two dimensional setting. We study critical sets of the isoperimetric problem and stationary sets for the flow (1.1). A set of finite perimeter $E$ is critical for the isoperimetric problem if its distributional mean curvature is constant.

We define stationary sets for the equation (1.1) as follows.

Definition 3.1. Assume that the forcing term $f$ in (1.1) is constant, i.e., $f \equiv c_{0}$. A set of finite perimeter $E_{0}$ is stationary if for any flat flow $\left(E_{t}\right)_{t}$ starting from $E_{0}$ it holds

$$
\sup _{0 \leq t \leq T}\left|E_{t} \Delta E_{0}\right|=0
$$

for every $T>0$.

We begin by proving the sharp quantitative version of the Alexandrov's theorem in the plane.

Lemma 3.2. Let $M>0$ and let $E \subset \mathbb{R}^{2}$ be $C^{2}$-regular with $P(E) \leq M$. There exist a constant $C_{M}$ and points $x_{1}, x_{2}, \ldots, x_{N}$, with $\left|x_{i}-x_{j}\right|>2$, such that for $F=\cup_{i=1}^{N} D_{1}\left(x_{i}\right)$ it holds

$$
\sup _{x \in E \Delta F} d_{\partial F}(x) \leq C_{M}\left\|k_{E}-1\right\|_{L^{1}(\partial E)}
$$

and

$$
|P(E)-2 \pi N| \leq C_{M}\left\|k_{E}-1\right\|_{L^{1}(\partial E)} .
$$

Moreover, there exists $\varepsilon_{0}>0$ such that if $\left\|k_{E}-1\right\|_{L^{2}(\partial E)} \leq \varepsilon_{0}$ then $E$ is $C^{1}$-diffeomorphic to the disjoint union of $N$ disks.

Proof. Assume that $\left\|k_{E}-1\right\|_{L^{1}(\partial E)} \geq \varepsilon_{0}$ for a small $\varepsilon_{0}$ to be chosen later. Since $\left\|k_{E}\right\|_{L^{1}(\partial E)}<$ $\infty, E$ has finitely many connected components $E_{i}, i=1, \ldots, N$. If $P(E) \geq 2 \pi N$, then $|P(E)-2 \pi N| \leq M$, hence (3.2) follows with a sufficiently large constant. Otherwise, using Gauss-Bonnet theorem,

$$
2 \pi N-P(E) \leq \sum_{i=1}^{N} \int_{\partial E_{i}}\left(\left|k_{E}\right|-1\right) d \mathcal{H}^{1} \leq\left\|k_{E}-1\right\|_{L^{1}(\partial E)},
$$


hence (3.2) follows with $C_{M}=1$. Since $P\left(E_{i}\right) \leq M$ for every $i$, there exist points $x_{i}$ such that $E_{i} \subset D_{M}\left(x_{i}\right)$. Therefore $\sup _{x \in E_{i} \Delta D_{1}\left(x_{i}\right)} d_{\partial D_{1}\left(x_{i}\right)}(x)$ is smaller than $M$. Hence $\sup _{x \in E \Delta F} d_{\partial F}(x) \leq M$ and (3.1) holds with a sufficiently large constant.

Assume now that $\left\|k_{E}-1\right\|_{L^{1}(\partial E)} \leq \varepsilon_{0}$ for a small $\varepsilon_{0}$. Let us fix a component $E_{i}$ of $E$ and denote $l=P\left(E_{i}\right)$. Let us first prove that there is $x_{i}$ such that

$$
\sup _{x \in E \Delta D_{1}\left(x_{i}\right)} d_{\partial D_{1}\left(x_{i}\right)}(x) \leq C\left\|k_{E}-1\right\|_{L^{1}(\partial E)} \quad \text { and } \quad|l-2 \pi| \leq\left\|k_{E}-1\right\|_{L^{1}\left(\partial E_{i}\right)} .
$$

It is not difficult to see that the claim follows from (3.3).

We claim first that $E_{i}$ is simply connected. Indeed, let $\Gamma_{0}$ be the outer component of $\partial E_{i}$ for which it holds $\int_{\Gamma_{0}} k_{E} d \mathcal{H}^{1}=2 \pi$. Then it follows from $\left\|k_{E}-1\right\|_{L^{1}(\partial E)} \leq \varepsilon_{0}$ that

$$
2 \pi-\mathcal{H}^{1}\left(\Gamma_{0}\right)=\int_{\Gamma_{0}}\left(k_{E}-1\right) d \mathcal{H}^{1} \leq \int_{\partial E}\left|k_{E}-1\right| d \mathcal{H}^{1} \leq \varepsilon_{0}
$$

This yields $P\left(E_{i}\right) \geq \mathcal{H}^{1}\left(\Gamma_{0}\right) \geq 2 \pi-\varepsilon_{0}$. Then

$$
\int_{\partial E_{i}} k_{E} d \mathcal{H}^{1}=\int_{\partial E_{i}}\left(k_{E}-1\right) d \mathcal{H}^{1}+P\left(E_{i}\right) \geq P\left(E_{i}\right)-\int_{\partial E}\left|k_{E}-1\right| d \mathcal{H}^{1} \geq 2 \pi-2 \varepsilon_{0} .
$$

Therefore when $\varepsilon_{0}<\pi$ we conclude that $\int_{\partial E_{i}} k_{E} d \mathcal{H}^{1}$ is positive. Since $E_{i}$ is connected, this implies that it is simply connected.

Since the boundary $\partial E_{i}$ is connected we may parametrize it by unit speed curve $\gamma:[0, l] \rightarrow$ $\mathbb{R}^{2}, \gamma(s)=(x(s), y(s))$ with counterclockwise orientation. Define $\theta(s):=\int_{0}^{s} k_{E}(\gamma(\tau)) d \tau$ so that $\theta(0)=0$ and $\theta(l)=2 \pi$. Then

$$
|\theta(s)-s| \leq\left\|k_{E}-1\right\|_{L^{1}\left(\partial E_{i}\right)} \quad \text { for all } s \in[0, l] .
$$

In particular, for $s=l(3.4)$ implies

$$
|\theta(l)-l|=|2 \pi-l| \leq\left\|k_{E}-1\right\|_{L^{1}\left(\partial E_{i}\right)}
$$

which is the second inequality in (3.3).

By possibly rotating the set $E$ we have

$$
x^{\prime}(s)=-\sin \theta(s) \quad \text { and } \quad y^{\prime}(s)=\cos \theta(s) .
$$

In particular, (3.4) implies

$$
\left|x^{\prime}(s)+\sin s\right| \leq\left\|k_{E}-1\right\|_{L^{1}\left(\partial E_{i}\right)} \quad \text { and } \quad\left|y^{\prime}(s)-\cos s\right| \leq\left\|k_{E}-1\right\|_{L^{1}\left(\partial E_{i}\right)}
$$

for all $s \in[0, l]$. Therefore there are numbers $a$ and $b$ such that

$$
|x(s)-a-\cos s| \leq C\left\|k_{E}-1\right\|_{L^{1}\left(\partial E_{i}\right)} \quad \text { and } \quad|y(s)-b-\sin s| \leq C\left\|k_{E}-1\right\|_{L^{1}\left(\partial E_{i}\right)}
$$

for all $s \in[0, l]$. Therefore we obtain from $|l-2 \pi| \leq\left\|k_{E}-1\right\|_{L^{1}(\partial E)}$ that

$|x(s)-a-\cos (2 \pi s / l)| \leq C\left\|k_{E}-1\right\|_{L^{1}\left(\partial E_{i}\right)} \quad$ and $\quad|y(s)-b-\sin (2 \pi s / l)| \leq C\left\|k_{E}-1\right\|_{L^{1}\left(\partial E_{i}\right)}$, which gives the first inequality in (3.3) for $x_{i}=(a, b)$.

Note that from (3.5) it follows that if $\left\|k_{E}-1\right\|_{L^{2}(\partial E)}$ is small, then $\gamma(s)$ is close in $C^{1, \alpha}(0, l)$ to the parametrization $(a+\cos (2 \pi s / l), b+\sin (2 \pi s / l))$ of $\partial D_{1}\left(x_{i}\right)$. Hence $E_{i}$ is $C^{1, \alpha}$-close to $D_{1}\left(x_{i}\right)$.

The following lemma is based on a comparison argument. 
Lemma 3.3. Assume $E_{0} \subset \mathbb{R}^{2}$ is $C^{2}$-regular set with $P\left(E_{0}\right) \leq M$ and let $\left(E_{t}^{h}\right)_{t}$ be the approximate flat flow starting from $E_{0}$. If $E_{0}$ is close to a disjoint union of $N$ disks with radius one, i.e., there exist $F=\cup_{i=1}^{N} D_{1}\left(x_{i}\right)$, with $\left|x_{i}-x_{j}\right| \geq 2$ for $i \neq j$, such that

$$
\sup _{x \in E_{t}^{h} \Delta F} d_{\partial F}(x) \leq \delta,
$$

then for $\delta>0$ small enough it holds

$$
\sup _{x \in E_{t}^{h} \Delta F} d_{\partial F}(x) \leq 5 \delta^{1 / 4} \quad \text { for all } t \in(0, \sqrt{\delta})
$$

for all $h>0$ small.

Proof. Let $F$ be the union of disks as in the assumption and define

$$
F_{-}:=\left\{x \in F: d_{\mathbb{R}^{2} \backslash F}(x)>\delta^{1 / 4}\right\} \quad \text { and } \quad F_{+}:=\left\{x \in \mathbb{R}^{2}: d_{F}(x)<\sqrt{\delta}\right\} .
$$

Then clearly $F_{-} \subset F \subset F_{+}$and by the assumption $\sup _{x \in E_{0} \Delta F} d_{\partial F}(x) \leq \delta$ it holds $F_{-} \subset E_{0} \subset$ $F_{+}$.

Let $\left(F_{t}^{h}\right)_{t}$ be the approximate flat flow with the constant forcing term $f=-\Lambda$, where $\Lambda:=C_{0}+1$, with $C_{0}$ as in (1.2), starting from $F_{-}$. Then by Proposition 2.1 it holds $F_{t}^{h} \subset E_{t}^{h}$ for all $t>0$. Note that $F_{-}$is a union of disks with radius $R=1-\delta^{1 / 4}$ and with positive distance to each other. It is easy to see that $\left(F_{t}^{h}\right)_{t}$ is decreasing, i.e., $F_{t}^{h} \subset F_{s}^{h}$ for $t>s$ and therefore it is enough to study the evolution of a one single disk $D_{R}$, because the flow $\left(F_{t}^{h}\right)_{t}$ is the union of them. If now $\left(\tilde{F}_{t}^{h}\right)$ is the approximate flat flow starting from $D_{R}$ with the forcing term $f=-\Lambda$ then it is not difficult to see that for $t \in(k h,(k+1) h]$ the set $\tilde{F}_{t}^{h}$ is a concentric disk with radius $r_{k+1}$ and by the Euler-Lagrange equation (2.3) it holds

$$
\frac{r_{k+1}-r_{k}}{h}=-\frac{1}{r_{k+1}}-\Lambda \text {. }
$$

Therefore, it holds

$$
r_{k+1}-r_{k} \geq-(\Lambda+2) h .
$$

for all $k=0,1,2, \ldots$ for which $r_{k+1} \geq 1 / 2$. By adding this over $k=0,1, \ldots, K$ with $\sqrt{\delta} / h \leq K \leq 2 \sqrt{\delta} / h$ and recalling that $r_{0}=R=1-\delta^{1 / 4}$ we obtain

$$
r_{K} \geq r_{0}-2 \sqrt{\delta}(\Lambda+2) \geq 1-2 \delta^{1 / 4},
$$

when $\delta$ is small. This implies $\sup _{x \in D_{R} \backslash \tilde{F}_{t}^{h}} d_{\partial D_{R}}(x) \leq 2 \delta^{1 / 4}$ for $t \in(0, \sqrt{\delta})$ and thus by the previous discussion

$$
\sup _{x \in F \backslash F_{t}^{h}} d_{\partial F}(x) \leq 2 \delta^{1 / 4} \quad \text { for } t \in(0, \sqrt{\delta}) .
$$

Since $F_{t}^{h} \subset E_{t}^{h}$ we have

$$
\sup _{x \in F \backslash E_{t}^{h}} d_{\partial F}(x) \leq 2 \delta^{1 / 4} \quad \text { for } t \in(0, \sqrt{\delta}) .
$$

We need yet to show that

$$
\sup _{x \in E_{t}^{h} \backslash F} d_{\partial F}(x) \leq 5 \delta^{1 / 4} \quad \text { for } t \in(0, \sqrt{\delta}) .
$$

Denote $\Gamma=\left\{x \in \mathbb{R}^{2} \backslash F: d_{\partial F}(x)=5 \delta^{1 / 4}\right\}$. Fix $x \in \Gamma$ and denote the disk $D_{r}(x)$ with $r=4 \delta^{1 / 4}$. Then by $E_{0} \subset F_{+}$and $D_{r}(x) \subset \mathbb{R}^{2} \backslash F_{+}$we have $E_{0} \subset \mathbb{R}^{2} \backslash D_{r}(x)$ if $\delta$ is sufficiently small. Let $\left(G_{t}^{h}\right)_{t}$ be the approximate flat flow starting from $D_{r}(x)$ with the constant forcing 
term $f=-\Lambda$. Arguing as above we deduce that for $t \in(k h,(k+1) h]$ the set $G_{t}^{h}$ is disk with radius $r_{k+1}$, i.e., $G_{t}^{h}=D_{r_{k+1}}(x)$ and

$$
\frac{r_{k+1}-r_{k}}{h}=-\frac{1}{r_{k+1}}-\Lambda \geq-\delta^{-1 / 4}-\Lambda
$$

for $k=0,1, \ldots$ for which $r_{k+1} \geq \delta^{1 / 4}$. By adding this over $k=0,1, \ldots, K$ with $\sqrt{\delta} / h \leq K \leq$ $2 \sqrt{\delta} / h$ and recalling that $r_{0}=r=4 \delta^{1 / 4}$ we obtain

$$
r_{K} \geq r_{0}-2 \sqrt{\delta}\left(\delta^{-1 / 4}+\Lambda\right) \geq 4 \delta^{1 / 4}-3 \delta^{1 / 4}=\delta^{1 / 4},
$$

when $\delta$ is small. In other words $D_{\delta^{1 / 4}}(x) \subset G_{t}^{h}$ for all $t \in(0, \sqrt{\delta})$. Since $E_{0} \subset \mathbb{R}^{2} \backslash D_{r}(x)$ Proposition 2.1 yields

$$
E_{t}^{h} \subset \mathbb{R}^{2} \backslash G_{t}^{h} \subset \mathbb{R}^{2} \backslash D_{\delta^{1 / 4}}(x)
$$

for all $0<t \leq \sqrt{\delta}$. By repeating the same argument for all $x \in \Gamma$ we conclude that the flow $E_{t}^{h}$ does not intersect $\Gamma$ for any $t \in(0, \sqrt{\delta})$. This implies (3.7).

In the next lemma we show that if $E_{0}$ is stationary then necessarily it is a disjoint union of disks, i.e., a critical set of the isoperimetric problem.

Lemma 3.4. Assume $E_{0} \subset \mathbb{R}^{2}$ is a bounded set of finite perimeter. If $E_{0}$ is stationary according to Definition 3.1 then it is a disjoint union of disks with equal radii.

Proof. Let us fix $T>1$ and $\varepsilon>0$ and let $\left(E_{t}^{h}\right)_{t}$ be an approximate flat flow starting from $E_{0}$. Then for any $\delta>0$ it holds by Definition 3.1 and by Proposition 2.3 that

$$
\sup _{h<t \leq T}\left|E_{t}^{h} \Delta E_{0}\right| \leq \delta
$$

for small $h$. Now the forcing term satisfies trivially the assumption (1.4) and therefore the left hand side of (2.8) is always zero. Then from the proof of Proposition 2.4 (i) we get that for every $h$ sufficiently small

$$
c \int_{2 h}^{T} \int_{\partial E_{t}^{h}}\left(k_{E_{t}^{h}}-c_{0}\right)^{2} d \mathcal{H}^{1} d t+\mathcal{E}\left(E_{T}^{h}\right) \leq \mathcal{E}\left(E_{h}^{h}\right) .
$$

Recall that $\mathcal{E}(E)=P(E)-c_{0}|E|$. By (3.8) it holds $\left|E_{T}^{h} \Delta E_{h}^{h}\right| \leq 2 \delta<\frac{\varepsilon}{c_{0}}$. Therefore we have

$$
c \int_{2 h}^{T} \int_{\partial E_{t}^{h}}\left(k_{E_{t}^{h}}-c_{0}\right)^{2} d \mathcal{H}^{1} d t+P\left(E_{T}^{h}\right) \leq P\left(E_{h}^{h}\right)+\varepsilon .
$$

Finally by (2.6) and (3.8) we obtain

$$
P\left(E_{h}^{h}\right) \leq P\left(E_{0}\right)+c_{0}\left(\left|E_{h}^{h}\right|-\left|E_{0}\right|\right) \leq P\left(E_{0}\right)+c_{0} \delta \leq P\left(E_{0}\right)+\varepsilon .
$$

Hence,

$$
c \int_{2 h}^{T} \int_{\partial E_{t}^{h}}\left(k_{E_{t}^{h}}-c_{0}\right)^{2} d \mathcal{H}^{1} d t+P\left(E_{T}^{h}\right) \leq P\left(E_{0}\right)+2 \varepsilon .
$$

By (3.8) it holds $\left|E_{T}^{h} \Delta E_{0}\right| \leq \delta$. Therefore by the lower semicontinuity of the perimeter it holds $P\left(E_{0}\right) \leq P\left(E_{T}^{h}\right)+\varepsilon$ when $\delta$ and $h$ are small. Therefore we have

$$
c \int_{2 h}^{T} \int_{\partial E_{t}^{h}}\left(k_{E_{t}^{h}}-c_{0}\right)^{2} d \mathcal{H}^{1} d t \leq 3 \varepsilon .
$$


By the mean value theorem there is $t<T$ such that

$$
\left\|k_{E_{t}^{h}}-c_{0}\right\|_{L^{2}} \leq C \sqrt{\varepsilon} .
$$

Since by Proposition $2.4 \sup _{t>0} P\left(E_{t}^{h}\right) \leq M$ for some $M$ independent of $h$, from the previous inequality and from Lemma 3.2 it follows that there are points $x_{1}, \ldots, x_{N}$, with $\left|x_{i}-x_{j}\right| \geq 2 r$, where $r=\frac{1}{c_{0}}$, such that for the set $F=\cup_{i=1}^{N} D_{r}\left(x_{i}\right)$ it holds

$$
\sup _{x \in E_{t}^{h} \Delta F} d_{\partial F}(x) \leq C \sqrt{\varepsilon} .
$$

Thus by (3.8) it holds

$$
\left|E_{0} \Delta F\right| \leq C \sqrt{\varepsilon}
$$

Note that the points $x_{i}$ might depend on $t$ and on $h$ but the radius $r$ and the number of disks $N$ does not. Therefore we conclude that the set $E_{0}$ is arbitrarily close to a union of essentially disjoint disks. This implies that the set $E_{0}$ itself is a union of essentially disjoint disks with radii $r=\frac{1}{c_{0}}$.

Proof of Theorem 1.1. Without loss of generality we may assume that

$$
E_{0}=D_{1}\left(-e_{1}\right) \cup D_{1}\left(e_{1}\right)
$$

where $e_{1}=(1,0)$. Let us now fix a small $h>0$ and consider the minimization problem (2.1) which gives a sequence of sets $\left(E^{h, k}\right)_{k=1}^{\infty}$ and thus an approximate flat flow $\left(E_{t}^{h}\right)_{t}$.

Let us fix $\varepsilon_{0}>0$. Then for $\delta$ small enough we have by Lemma 3.3 that for $k \leq \frac{\delta}{h}$ it holds

$$
\left(D_{1-\varepsilon_{0}}\left(-e_{1}\right) \cup D_{1-\varepsilon_{0}}\left(e_{1}\right)\right) \subset E^{h, k} \subset\left(D_{1+\varepsilon_{0}}\left(-e_{1}\right) \cup D_{1+\varepsilon_{0}}\left(e_{1}\right)\right),
$$

when $h$ is small. Moreover, by Lemma 2.2 it holds

$$
\left(D_{1-C_{1} \sqrt{h}}\left(-e_{1}\right) \cup D_{1-C_{1} \sqrt{h}}\left(e_{1}\right)\right) \subset E^{h, 1} .
$$

Let us improve the above estimate and show that the set $E^{h, 1}$ contains a large simply connected set. To be more precise, we denote the rectangle $R_{\eta}^{h}=\left(-2 C_{1} \sqrt{h}, 2 C_{1} \sqrt{h}\right) \times$ $\left(-\eta h^{1 / 4}, \eta h^{1 / 4}\right)$ and prove that for $\eta>0$ small, independent of $h$, it holds

$$
\left(D_{1-C_{1} \sqrt{h}}\left(-e_{1}\right) \cup D_{1-C_{1} \sqrt{h}}\left(e_{1}\right)\right) \cup R_{\eta}^{h} \subset E^{h, 1} .
$$

We argue by contradiction assuming that $\partial E^{h, 1} \cap\left(-2 C_{1} \sqrt{h}, 2 C_{1} \sqrt{h}\right) \times\left(-\eta h^{1 / 4}, \eta h^{1 / 4}\right)$ is non-empty. We denote the rectangle $R_{3 \eta}^{h}=\left(-2 C_{1} \sqrt{h}, 2 C_{1} \sqrt{h}\right) \times\left(-3 \eta h^{1 / 4}, 3 \eta h^{1 / 4}\right)$ and define

$$
\tilde{E}^{h, 1}=E^{h, 1} \cup R_{3 \eta}^{h} .
$$

By the contradiction assumption the length of the curve $\partial E^{h, 1} \cap R_{3 \eta}^{h}$ is greater than $2 \eta h^{1 / 4}$. We have then the following estimate

$$
P\left(\tilde{E}^{h, 1}\right) \leq P\left(E^{h, 1}\right)-\eta h^{1 / 4},
$$

when $h$ is small. We also have

(i) ||$\tilde{E}^{h, 1}|-| E^{h, 1}|| \leq\left|R_{3 \eta}^{h}\right|=24 C_{1} \eta h^{\frac{3}{4}}$

(ii) $\left|\bar{d}_{E_{0}}(x)\right| \leq 10 \eta^{2} \sqrt{h}$ for all $x \in R_{3 \eta}^{h} \backslash E_{0}$. 
It follows from (i), (ii) and $\sup _{t}|f(t)| \leq C_{0}$ that

$$
\begin{aligned}
\frac{1}{h} \int_{\tilde{E}^{h, 1}} & \bar{d}_{E_{0}} d x+\bar{f}(h)\left|\tilde{E}^{h, 1}\right| \\
& \leq \frac{1}{h} \int_{E^{h, 1}} \bar{d}_{E_{0}} d x+\bar{f}(h)\left|E^{h, 1}\right|+\frac{1}{h} \int_{R_{3 \eta}^{h} \backslash E_{0}} \bar{d}_{E_{0}} d x+C_{0}|| \tilde{E}^{h, 1}|-| E^{h, 1}|| \\
& \leq \frac{1}{h} \int_{E^{h, 1}} \bar{d}_{E_{0}} d x+\bar{f}(h)\left|E^{h, 1}\right|+C \eta^{3} h^{1 / 4}
\end{aligned}
$$

when $h$ is small. Therefore using (3.11) and the above inequality we may estimate

$$
\mathcal{F}\left(\tilde{E}^{h, 1} ; E_{0}\right) \leq \mathcal{F}\left(E^{h, 1} ; E_{0}\right)-\eta h^{1 / 4}+C \eta^{3} h^{1 / 4}<\mathcal{F}\left(E^{h, 1} ; E_{0}\right)
$$

when $\eta>0$ is small enough. This contradicts the minimality of $E^{h, 1}$ and we obtain (3.10).

We continue by constructing a barrier set $G_{h}$ (see Figure 3.1) and prove that $G_{h} \subset E^{h, k}$ for every $k \leq \delta / h$. For $h \geq 0$ we define $\varphi_{h}:\left(-3 \varepsilon_{0}, 3 \varepsilon_{0}\right) \rightarrow \mathbb{R}$ as

$$
\varphi_{h}(s)=3 \varepsilon_{0}-\sqrt{9 \varepsilon_{0}^{2}-s^{2}}+h
$$

and define the set

$$
G_{\varphi_{h}}:=\left\{\left(x_{1}, x_{2}\right) \in \mathbb{R}^{2}: x_{1} \in\left(-3 \varepsilon_{0}, 3 \varepsilon_{0}\right),\left|x_{2}\right|<\varphi_{h}\left(x_{1}\right)\right\},
$$

which is 'the neck'. We define the barrier set as

$$
G_{h}=\left(D_{1-2 \varepsilon_{0}}\left(-e_{1}\right) \cup D_{1-2 \varepsilon_{0}}\left(e_{1}\right)\right) \cup G_{\varphi_{h}} .
$$

The barrier set $G_{h}$ is open and connected and we have the estimate on the curvature at the neck

$$
\text { for every } x \in \partial G_{h} \backslash\left(\bar{D}_{1-2 \varepsilon_{0}}\left(-e_{1}\right) \cup \bar{D}_{1-2 \varepsilon_{0}}\left(e_{1}\right)\right) \text { it holds } k_{G_{0}}(x)=-\frac{1}{3 \varepsilon_{0}} \text {. }
$$

Moreover, we notice that when $h$ is small then by (3.10) it holds

$$
G_{h} \subset E^{h, 1}
$$

In fact, (3.10) implies that

$$
\inf _{\mathbb{R}^{2} \backslash E^{h, 1}} d_{G_{h}}(x) \geq c h^{1 / 4}
$$

for small $c>0$.

Let us define

$$
\rho_{k}:=\inf _{\mathbb{R}^{2} \backslash E^{h, k}} d_{G_{h}}(x)
$$

for $k=1,2, \ldots$ and $\rho_{0}:=0$. We claim that for every $k \leq \frac{\delta}{h}$ it holds

$$
\rho_{k+1}-\rho_{k} \geq 2 h \quad \text { or } \quad \rho_{k+1} \geq \frac{\varepsilon_{0}}{2}
$$

when $h$ is small.

We prove (3.14) by induction and notice that for $k=0$ the inequality (3.14) is already proven since (3.13) implies $\rho_{1} \geq c h^{1 / 4}$. Let us assume that (3.14) holds for $k-1$ and prove it for $k$. Let us assume that $\rho_{k+1}<\frac{\varepsilon_{0}}{2}$. The induction assumption and $\rho_{1} \geq c h^{1 / 4}$ yields $\rho_{k} \geq c h^{1 / 4}$. On the other hand by Lemma 2.2 it holds $\sup _{E^{h, k+1} \Delta E^{h, k}} d_{\partial E^{h, k}} \leq C_{1} \sqrt{h}$ and therefore $\rho_{k+1}>0$. 


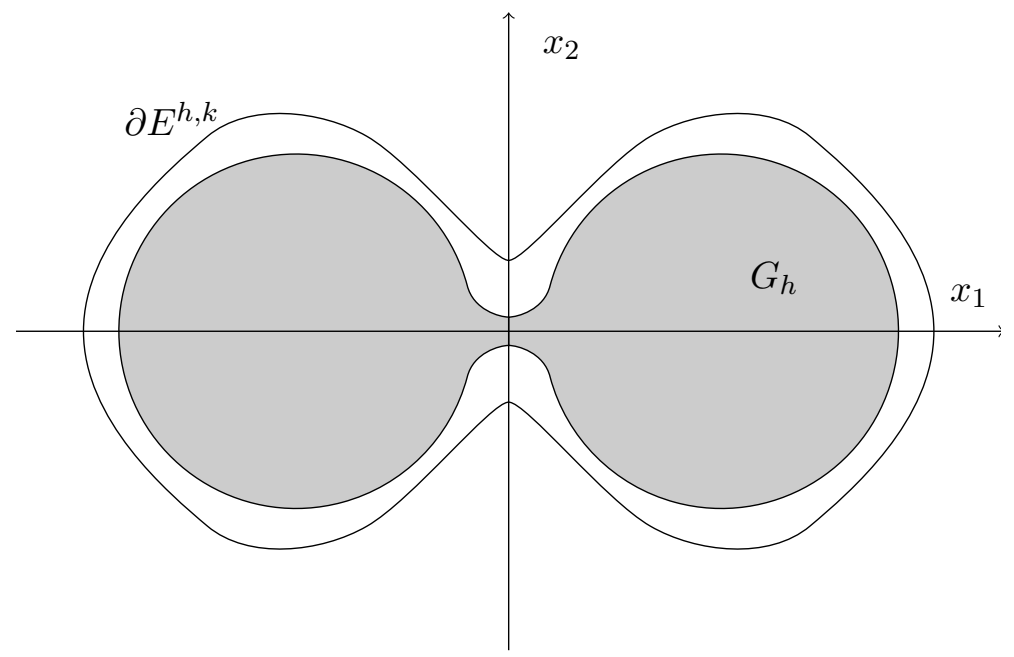

FIgURE 3.1. The boundary of $\partial E^{h, k}$ lies outside of the barrier set $G_{h}$.

Let $x_{k+1} \in \partial E^{h, k+1}$ and $y_{k+1} \in \partial G_{h}$ be such that $\left|x_{k+1}-y_{k+1}\right|=\min _{x \in \partial E^{h, k+1}} d_{G_{h}}(x)=$ $\rho_{k+1}$. By (3.9) it holds $x_{k+1} \notin D_{1-\varepsilon_{0}}\left(-e_{1}\right) \cup D_{1-\varepsilon_{0}}\left(e_{1}\right)$ and therefore by $\rho_{k+1}<\frac{\varepsilon_{0}}{2}$ we have

$$
y_{k+1} \in \partial G_{h} \backslash\left(\bar{D}_{1-2 \varepsilon_{0}}\left(-e_{1}\right) \cup \bar{D}_{1+2 \varepsilon_{0}}\left(e_{1}\right)\right) .
$$

Then (3.12) yields

$$
k_{G_{h}}\left(y_{k+1}\right)=-\frac{1}{3 \varepsilon_{0}} .
$$

Since $x_{k+1}$ is a point of minimal distance $k_{E^{h, k+1}}\left(x_{k+1}\right) \leq k_{G_{h}}\left(y_{k+1}\right)=-\frac{1}{3 \varepsilon_{0}}$. By taking $\varepsilon_{0}$ smaller, if needed, we have by the Euler-Lagrange equation (2.3) and by $\sup _{t>0}|f(t)| \leq C_{0}$ that

$$
\frac{\bar{d}_{E^{h, k}}\left(x_{k+1}\right)}{h}=-k_{E^{h, k+1}}\left(x_{k+1}\right)+\bar{f}(k h) \geq \frac{1}{3 \varepsilon_{0}}-C_{0} \geq 2 .
$$

The inequality (3.15) and $G_{h} \subset E^{h, k}$ imply that $x_{k+1} \notin E^{h, k}$ and $y_{k+1} \in E^{h, k}$. Thus there is a point $z_{k+1}$ on the segment $\left[x_{k+1}, y_{k+1}\right]$ such that $z_{k+1} \in \partial E^{h, k}$. Since $y_{k+1} \in \partial G_{h}$ it holds $\left|z_{k+1}-y_{k+1}\right| \geq \rho_{k}$ and by (3.15) we have $\left|x_{k+1}-z_{k+1}\right| \geq \bar{d}_{E^{h, k}}\left(x_{k+1}\right) \geq 2 h$. Therefore because $z_{k+1}$ is on the segment $\left[x_{k+1}, y_{k+1}\right]$ we have

$$
\rho_{k+1}=\left|x_{k+1}-y_{k+1}\right|=\left|x_{k+1}-z_{k+1}\right|+\left|z_{k+1}-y_{k+1}\right| \geq 2 h+\rho_{k} .
$$

Thus we have (3.14).

Let us conclude the proof. By adding (3.14) together for $k=0,1,2, \ldots, K$ with $K \leq \delta / h$ we deduce that for $\delta$ small it holds

$$
\inf _{\mathbb{R}^{2} \backslash E_{t}^{h}} d_{G_{h}}(x) \geq t \quad \text { for all } t \in(h, \delta] .
$$

In particular $G_{h} \subset E_{t}^{h}$. Let $h_{l} \rightarrow 0$ be any sequence such that $\sup _{h_{l}<t \leq \delta}\left|E_{t}^{h_{l}} \Delta E_{t}\right| \rightarrow 0$, see Proposition 2.3. By (3.16) we get

$$
\inf _{\mathbb{R}^{2} \backslash E_{t}} d_{G_{0}}(x) \geq t \quad \text { for all } t \in(0, \delta] .
$$


This inequality implies, in particular, that $E_{t}$ contains a ball centered at the origin with radius $t$ for all $t \in(0, \delta)$ and that

$$
\left|E_{t} \backslash E_{0}\right| \geq c t^{3}
$$

This is the second statement of Theorem 1.1. The inequality (3.16) implies that

$$
\left\{x \in \mathbb{R}^{2}: d_{G_{h}}(x)<t\right\} \subset E_{t}^{h} \quad \text { for all } t \in(h, \delta] .
$$

Passing to the limit as above, along the subsequence $h_{l}$, we deduce

$$
\left\{x \in \mathbb{R}^{2}: d_{G_{0}}(x)<t\right\} \subset E_{t} \quad \text { for all } t \in(0, \delta] .
$$

The first claim follows from the fact that $\left\{x \in \mathbb{R}^{2}: d_{G_{0}}(x)<t\right\}$ is open and simply connected.

We conclude this section by explaining how Corollary 1.2 follows from Theorem 1.1.

Proof of Theorem 1.2. First, it is easy to see that if $E_{0}$ is a union of disks with equal radius and with positive distance to each other, then $E_{0}$ is stationary according to the Definition 3.1. If $E_{0}$ is stationary then by Lemma 3.4 it is critical, i.e., finite union of essentially disjoint disks $D_{r}\left(x_{i}\right)$, with $r=1 / c_{0}$ and $i=1, \ldots, N$. We need to show if $i \neq j$ then $\left|x_{i}-x_{j}\right|>2 / c_{0}$. If by contradiction there are two tangential disks, say $D_{r}\left(x_{1}\right)$ and $D_{r}\left(x_{2}\right)$, then we define $F_{0}=D_{r}\left(x_{1}\right) \cup D_{r}\left(x_{2}\right)$. Let $\left(E_{t}\right)_{t}$ be a flat flow starting from $E_{0}$ and let $h_{l} \rightarrow 0$ be a sequence such that $\left|E_{t}^{h_{l}} \Delta E_{t}\right| \rightarrow 0$ and $\left|F_{t}^{h_{l}} \Delta F_{t}\right| \rightarrow 0$, where $\left(F_{t}\right)_{t}$ is a flat flow starting from $F_{0}$ with forcing term $g=c_{0}-\varepsilon$. Then by Proposition $2.1 F_{t}^{h_{l}} \subset E_{t}^{h_{l}}$ for all $t>0$ and $h_{l}$, hence $F_{t} \subset E_{t}$. By Theorem 1.1 we have that there exist $\delta, c>0$ such that for all $t \in(0, \delta)$

$$
\left|F_{t} \backslash F_{0}\right| \geq c t^{3} \text {. }
$$

This implies

$$
\left|E_{t} \backslash E_{0}\right| \geq c t^{3}
$$

and therefore $E_{0}$ is not stationary.

\section{Proofs of Theorem 1.3 And Theorem 1.4}

Proof of Theorem 1.3. First, by scaling we may assume that $c_{0}=1$ in the assumption (1.4).

Let $\left(E_{t}^{h}\right)$ be an approximate flat flow which converges, up to a subsequence, to $\left(E_{t}\right)_{t}$. We simplify the notation and denote the converging subsequence again by $h$. From the assumption $\sup _{t>0}\left|E_{t}\right|=M$ and from Proposition 2.3 (iv) it follows that for every $T>0$ there is $h_{T}$ such that up to subsequence of $h$ it holds

$$
\sup _{0<t<T}\left|E_{t}^{h}\right| \leq 2 M \quad \text { for all } 0<h<h_{T} .
$$

Then Remark 2.5 yields that there exists a constant $\tilde{C}$ independent of $h$ and $T$ such that for $0<h<h_{T}$

$$
\sup _{0<t<T} P\left(E_{t}^{h}\right) \leq \tilde{C}
$$

The dissipation inequality in Proposition 2.4 and the above volume and perimeter bounds imply

$$
\int_{T_{0}}^{T} \int_{\partial E_{t}^{h}}\left(k_{E_{t}^{h}}-\bar{f}(t-h)\right)^{2} d \mathcal{H}^{1} d t \leq C
$$


for some $T_{0}>0$ for every $T>T_{0}$. Then the assumption (1.4) (recall that $c_{0}=1$ ) yields

$$
\int_{T_{0}}^{T} \int_{\partial E_{t}^{h}}\left(k_{E_{t}^{h}}-1\right)^{2} d \mathcal{H}^{1} d t \leq C .
$$

for some $T_{0}$ large and for every $T>T_{0}$ and $0<h<h_{T}$. In particular, if we denote $I_{j}=\left[(j-1)^{2}, j^{2}\right]$ for $j=1,2, \ldots, k<\sqrt{T}$ then it holds

$$
\int_{I_{j}} \int_{\partial E_{t}^{h}}\left(k_{E_{t}^{h}}-1\right)^{2} d \mathcal{H}^{1} d t \leq C
$$

for $j$ large. Let us fix a small $\varepsilon>0$. From the previous inequality we obtain that there exists $j_{\varepsilon}$ such that, if $j_{\varepsilon} \leq j \leq \sqrt{T}$ and $0<h<h_{T}$ there exists $T_{h, j}$ such that

$$
(j-1)^{2} \leq T_{h, j} \leq j^{2}, \quad \text { and } \quad\left\|k_{E_{T_{h, j}^{h}}^{h}}-1\right\|_{L^{2}\left(E_{T_{h, j}}^{h}\right)} \leq \varepsilon .
$$

We deduce by Lemma 3.2 that the set $E_{T_{h, j}}^{h}$ is close to a disjoint union of $N_{h, j}$ disks of radius one. Since the measures of $E_{T_{h, j}}^{h}$ are uniformly bounded, we conclude that there is $N_{0}$ such that $N_{h, j} \leq N_{0}$. Moreover, we have by Lemma 3.2 that

$$
\left|P\left(E_{T_{h, j}}^{h}\right)-2 \pi N_{h, j}\right|+|| E_{T_{h, j}}^{h}\left|-\pi N_{h, j}\right| \leq C \varepsilon .
$$

This implies the following estimate for the energy $\mathcal{E}\left(E_{T_{h, j}}^{h}\right)=P\left(E_{T_{h, j}}^{h}\right)-\left|E_{T_{h, j}}^{h}\right|$,

$$
\left|\mathcal{E}_{T_{h, j}}\left(E_{T_{h, j}}^{h}\right)-\pi N_{h, j}\right| \leq C \varepsilon .
$$

In other words, at $T_{h, j}$ the energy has almost the value $\pi N_{h, j}$. Since the energy $\mathcal{E}_{T_{h, j}}\left(E_{T_{h, j}}^{h}\right)$ is asymptotically almost decreasing by Remark 2.5 , we deduce that the sequence of numbers $N_{h, j}$ is decreasing for $j$ large, i.e.,

$$
N_{h, j} \geq N_{h, j+1} \quad \text { for every } j_{\varepsilon} \leq j \leq \sqrt{T} .
$$

By letting $h \rightarrow 0$ we conclude by Proposition 2.3 (iv) and by standard diagonal argument that, by extracting another subsequence if needed, there is sequence of times $T_{j}$, with $j \geq j_{\varepsilon}$, such that $(j-1)^{2} \leq T_{j} \leq j^{2}$ and the set $E_{T_{j}}$ is close to $N_{j}$ many disjoint disks of radius one and that $N_{j} \geq N_{j+1}$ for every $j \geq j_{\varepsilon}$. This implies that, there is $j_{0} \geq j_{\varepsilon}$ and $N$ such that

$$
N_{j}=N \quad \text { for all } j \geq j_{0} .
$$

This means that every $E_{T_{j}}$, for $j \geq j_{0}$, is close in $L^{1}$-sense to disjoint union of exactly $N$ many disks of radius one. By the locally uniform $L^{1}$-convergence $\left(E_{t}^{h}\right)_{t} \rightarrow\left(E_{t}\right)$ for every $T>j_{0}^{2}$ we have

$$
N_{h, j}=N \quad \text { for } j_{0} \leq j \leq \sqrt{T},
$$

when $h$ is small. Therefore we conclude from (4.1) and from the dissipation inequality in Proposition 2.4 that for any $\delta>0$ there is $T_{\delta}$ such that for all $T>T_{\delta}$ it holds

$$
\int_{T_{\delta}}^{T} \int_{\partial E_{t}^{h}}\left(k_{E_{t}^{h}}-1\right)^{2} d \mathcal{H}^{1} d t \leq \delta^{3}
$$

when $h$ is small.

Let us fix $T>>T_{\delta}$ and denote by $J_{h} \subset\left(T_{\delta}, T\right)$ the set of times $t \in\left(T_{\delta}, T\right)$ for which

$$
\left\|k_{E_{t}^{h}}-1\right\|_{L^{2}\left(\partial E_{t}^{h}\right)} \geq \delta \text {. }
$$


Then by (4.4) it holds $\left|J_{h}\right| \leq \delta$. If $\delta$ is small enough, by Lemma 3.2, from (4.2), (4.3) and (2.10) we deduce that the sets $E_{t}^{h}$ satisfy

$$
\sup _{x \in E_{t}^{h} \Delta F_{t}^{h}} d_{\partial F_{t}^{h}}(x) \leq C \delta \quad \text { for all } t \in\left(T_{\delta}, T\right) \backslash J_{h},
$$

where $F_{t}^{h}=\cup_{i=1}^{N} D_{1}\left(x_{i}\right)$ with $\left|x_{i}-x_{j}\right| \geq 2$ for $i \neq j$. Note that the points $x_{i}$ may depend on $t$ and $h$.

We will show that for all $t \in\left(T_{\delta}+2 \delta, T\right)$ it holds

$$
\sup _{x \in E_{t}^{h} \Delta F_{t}^{h}} d_{\partial F_{t}^{h}}(x) \leq C \delta^{1 / 4}
$$

where $F_{t}^{h}$ is a union of $N$ disjoint disks as above. Let us fix $t_{0} \in\left(T_{\delta}, T\right) \backslash J_{h}$. By (4.5) we have

$$
\sup _{x \in E_{t_{0}}^{h} \Delta F_{t_{0}}^{h}} d_{\partial F_{t_{0}}^{h}}(x) \leq C \delta .
$$

We use Lemma 3.3 with $E_{0}=E_{t_{0}}^{h}$ to conclude

$$
\sup _{x \in E_{t}^{h} \Delta F_{t_{0}}^{h}} d_{\partial F_{t_{0}}^{h}}(x) \leq C \delta^{1 / 4} \quad \text { for all } t \in\left[t_{0}, t_{0}+\sqrt{\delta}\right) .
$$

This means that if we define

$$
I=\cup_{t \in\left(T_{\delta}, T\right) \backslash J_{h}}[t, t+\sqrt{\delta})
$$

then (4.6) holds for every $t \in I$. But since $\left|J_{h}\right| \leq \delta \leq \sqrt{\delta} / 2$, it easy to see that $\left(T_{\delta}+2 \delta, T\right) \subset I$. Thus (4.6) holds for every $t \in\left(T_{\delta}+2 \delta, T\right)$.

We have thus proved (4.6). The claim follows by letting $h \rightarrow 0$ and from Proposition 2.3.

We conclude the paper by proving Theorem 1.4. To this end we recall the Bonnesen symmetrization of a planar set.

Let $E \subset \mathbb{R}^{2}$ be a measurable set. The Bonnesen symmetrization of $E$ with respect to $x_{2}$-axis is the set $E^{*}$, with the property that for every $r>0$

$$
\mathcal{H}^{1}\left(\partial D_{r} \cap E^{*}\right)=\mathcal{H}^{1}\left(\partial D_{r} \cap E\right)
$$

and $\partial D_{r} \cap E^{*}$ is the union of two circular $\operatorname{arcs} \gamma_{r}^{+}$and $\gamma_{r}^{-}$with equal length, symmetric with respect to the $x_{2}$-axis and such that $\gamma_{r}^{-}$is obtained by reflecting $\gamma_{r}^{+}$with respect to the $x_{1}$-axis.

Clearly this symmetrization leaves the area unchanged. Moreover, if $E$ is a convex set, symmetric with respect to both coordinate axes, then $P\left(E^{*}\right) \leq P(E)$, see [6, Page 67] (see also [11]).

Let us prove that the Bonnesen symmetrization decreases the dissipation.

Lemma 4.1. Let $G \subset \mathbb{R}^{2}$ be invariant under Bonnesen symmetrization. Then for any measurable set $E \subset \mathbb{R}^{2}$ it holds

$$
\int_{E^{*}} \bar{d}_{G} d x \leq \int_{E} \bar{d}_{G} d x
$$

Proof. It is enough to prove that for every $r>0$ it holds

$$
\int_{\partial D_{r} \cap E^{*}} \bar{d}_{G} d \mathcal{H}^{1} \leq \int_{\partial D_{r} \cap E} \bar{d}_{G} d \mathcal{H}^{1}
$$




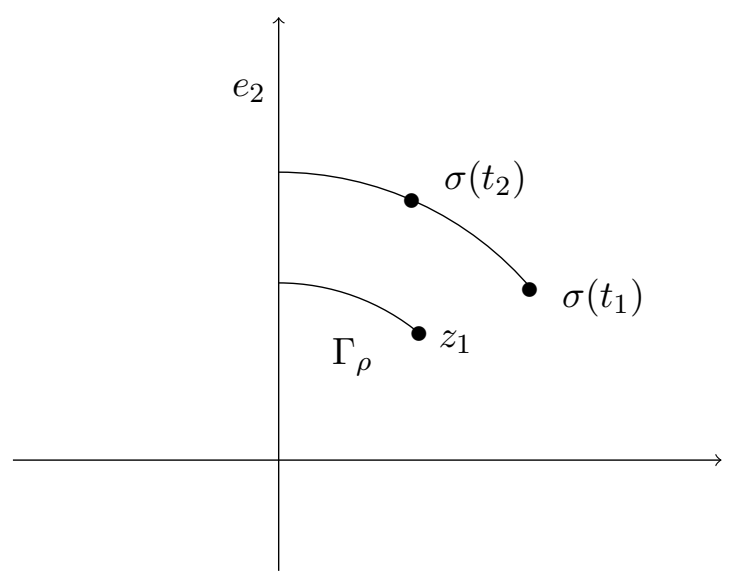

Figure 4.1. The point $\sigma\left(t_{2}\right)$ is closer to the $\operatorname{arc} \Gamma_{\rho}$ than $\sigma\left(t_{1}\right)$.

Let us fix $r>0$ and without loss of generality we may assume that $r=1$. Let $\sigma:[-\pi, \pi] \rightarrow \mathbb{R}^{2}$,

$$
\sigma(t)=\left[\begin{array}{l}
\cos (t) \\
\sin (t)
\end{array}\right] .
$$

Since $G$ is symmetric with respect to both coordinate axes, the function $t \mapsto \bar{d}_{G}(\sigma(t))$ is even and for every $t \in(0, \pi / 2)$ it holds $\bar{d}_{G}(\sigma(\pi-t))=\bar{d}_{G}(\sigma(t))$. We observe that (4.7) follows once we show that

$$
t \mapsto \bar{d}_{G}(\sigma(t)) \quad \text { is decreasing on } t \in(0, \pi / 2) .
$$

To this aim we fix $0<t_{1}<t_{2}<\pi / 2$. Let us assume that $\sigma\left(t_{1}\right) \in \mathbb{R}^{2} \backslash G$, the case $\sigma\left(t_{1}\right) \in G$ being similar. If $\sigma\left(t_{2}\right) \in G$ then trivially $\bar{d}_{G}\left(\sigma\left(t_{2}\right)\right) \leq 0 \leq \bar{d}_{G}\left(\sigma\left(t_{1}\right)\right)$. Let us thus assume that $\sigma\left(t_{2}\right) \in \mathbb{R}^{2} \backslash G$. Let $z_{1} \in \partial G$ be such that $\bar{d}_{G}\left(\sigma\left(t_{1}\right)\right)=\left|\sigma\left(t_{1}\right)-z_{1}\right|$ and let $\rho>0$ and $\theta_{1} \in(0, \pi / 2)$ be such that $z_{1}=\rho \sigma\left(\theta_{1}\right)$. We denote by $\Gamma_{\rho} \subset \partial D_{\rho}$ the arc with endpoints $\rho e_{2}$ and $z_{1}$, i.e.,

$$
\Gamma_{\rho}=\left\{\rho \sigma(t): t \in\left(\theta_{1}, \pi / 2\right)\right\} .
$$

Since $G$ is invariant under Bonnesen symmetrization we have $\Gamma_{\rho} \subset G$. But now since $t_{1}<$ $t_{2}<\pi / 2$ it clearly holds (see Figure 4.1 )

$$
\operatorname{dist}\left(\sigma\left(t_{2}\right), \Gamma_{\rho}\right) \leq \operatorname{dist}\left(\sigma\left(t_{1}\right), \Gamma_{\rho}\right)=\left|\sigma\left(t_{1}\right)-z_{1}\right|=\bar{d}_{G}\left(\sigma\left(t_{1}\right)\right) .
$$

Since $\Gamma_{\rho} \subset G$ we have

and the claim follows.

$$
\bar{d}_{G}\left(\sigma\left(t_{2}\right)\right) \leq \operatorname{dist}\left(\sigma\left(t_{2}\right), \Gamma_{\rho}\right)
$$


Proof of Theorem 1.4. Let $G$ be the ellipse

$$
G=\left\{\left(x_{1}, x_{2}\right) \in \mathbb{R}^{2}: a^{2} x_{1}^{2}+x_{2}^{2}<1\right\} \quad \text { with } a>1
$$

as in the assumption and let $\left(G_{t}\right)_{t}$ be the classical solution of the volume preserving mean curvature flow

$$
V_{t}=-k_{G_{t}}+\bar{k}_{G_{t}}
$$

starting from $G$. By [18], $\left(G_{t}\right)_{t}$ is well defined for all times, remains smooth and uniformly convex and converges exponentially fast to the disk $D_{\rho}$, where $\rho=\frac{1}{\sqrt{a}}$. Moreover $G_{t} \neq D_{\rho}$ for all $t>0$. Let us define $f(t):=\bar{k}_{G_{t}}$ which is therefore a smooth function and converges exponentially fast to $1 / \rho$. Note that then $f$ satisfies (1.4) for $c_{0}=1 / \rho$.

By the regularity of $E_{0}$ the flat flow $\left(E_{t}\right)_{t}$ with the forcing term $f$ starting from $E_{0}$ coincides with the unique classical solution provided by [18], see [9, Proposition 4.9]. Therefore, by the symmetry of $E_{0}$ we may conclude that

$$
E_{t}=\left(G_{t}-\rho e_{1}\right) \cup\left(G_{t}+\rho e_{1}\right)
$$

as long as the components $\left(G_{t}-\rho e_{1}\right)$ and $\left(G_{t}+\rho e_{1}\right)$ do not intersect each other. By the convexity of $G_{t}$, the components $G_{t}-\rho e_{1}$ and $G_{t}+\rho e_{1}$ do not intersect each other if the first one stays in the half-space $\left\{x_{1}<0\right\}$ and the latter in $\left\{x_{1}>0\right\}$. This is the same as to say that the flow $G_{t}$ does not exit the strip $\left\{-\rho<x_{1}<\rho\right\}$. Let us show this.

Assume that for $h>0$ the family of sets $\left(G_{t}^{h}\right)_{t}$ is an approximate flow obtained via (2.1) with the forcing term $f$ and starting from $G$. We now show that each $G_{t}^{h}$ is symmetric with respect to the coordinate axes and convex. Recall that the set $G^{h, 1}$ is chosen as a minimizer of the functional

$$
\mathcal{F}(E ; G)=P(E)+\frac{1}{h} \int_{E} \bar{d}_{G} d x-\bar{f}(h)|E| .
$$

It is well known that in any dimension the function in (4.9) admits a minimal and a maximal minimizer which are convex and, by uniqueness, symmetric with respect to both coordinate axes, see [3, Theorem 2]. However, in our two dimensional setting we can provide a simple self contained proof of this fact.

Given $E$, we set $E_{+}=\left\{x \in E: x_{1}>0\right\}$ and $E_{-}=\left\{x \in E: x_{1}<0\right\}$. By reflecting $E_{+}$and $E_{-}$with respect to the $x_{2}$-axis we obtain sets $E_{1}$ and $E_{2}$, which are symmetric with respect to the $x_{2}$-axis and satisfy

$$
\mathcal{F}\left(E_{1} ; G\right)+\mathcal{F}\left(E_{2} ; G\right) \leq 2 \mathcal{F}(E ; G) .
$$

Then there exists $i=1,2$ such that $\mathcal{F}\left(E_{i} ; G\right) \leq \mathcal{F}(E ; G)$. By repeating the same argument with respect to the $x_{1}$-direction we conclude that we may choose $G^{h, 1}$ symmetric with respect to both axes.

Let us show that $G^{h, 1}$ is convex. By the Euler-Lagrange equation (2.3) it holds

$$
\frac{\bar{d}_{G}}{h}=-k_{G^{h, 1}}+\bar{f}(h) \quad \text { on } \partial G^{h, 1}
$$

We claim that $\frac{\bar{d}_{G}}{h}(x) \leq \bar{f}(h)$ for all $x \in \partial G^{h, 1}$. Indeed, suppose $x_{0} \in \partial G^{h, 1}$ is the maximum of $\bar{d}_{G}$ on $\partial G^{h, 1}$. If $\bar{d}_{G}\left(x_{0}\right) \leq 0$, then trivially $\frac{\bar{d}_{G}}{h}\left(x_{0}\right) \leq \bar{f}(h)$ as $f \geq 0$. If $\bar{d}_{G}\left(x_{0}\right)>0$ then 
$x_{0} \notin G$ and since it is the furthest point from $G$ and $G$ is convex, it is easy to check that $k_{G^{h, 1}}\left(x_{0}\right) \geq 0$. Then by the Euler-Lagrange equation

$$
\frac{\bar{d}_{G}\left(x_{0}\right)}{h}=-k_{G^{h, 1}}\left(x_{0}\right)+\bar{f}(h) \leq \bar{f}(h) .
$$

Therefore $\bar{d}_{G} / h \leq \bar{f}(h)$ on $\partial G^{h, 1}$ and by the Euler-Lagrange equation

$$
k_{G^{h, 1}}=-\frac{\bar{d}_{G}}{h}+\bar{f}(h) \geq 0 \quad \text { on } \partial G^{h, 1} .
$$

Hence, $G^{h, 1}$ is convex.

We now apply to $G^{h, 1}$ the Bonnesen circular symmetrization with respect to the $x_{2}$-axis which, we recall, decreases the perimeter, preserves the area and decreases the dissipation term $\int_{G^{h, 1}} \bar{d}_{G} d x$, by Lemma 4.1. Therefore we may assume that $G^{h, 1}$ is invariant under the Bonnesen annular symmetrization with respect to the $x_{2}$-axis. By iterating the argument we deduce that the same holds for $G_{t}^{h}$ for all $t>0$. Letting $h \rightarrow 0$ we deduce that the same holds for the flat flow, and by the uniqueness for the classical solution $\left(G_{t}\right)_{t}$. Therefore for every $t>0$ and $r>0$ the intersection $G_{t} \cap \partial D_{r}$ is a union of two circular arcs with equal length which are both symmetric with respect to the $x_{2}$-axis.

Now if $G_{t}$ exits the strip $\left\{-\rho<x_{1}<\rho\right\}$, say at time $t_{0}$, then the intersection $G_{t_{0}} \cap \partial D_{\rho}$ contains the points $(-\rho, 0)$ and $(\rho, 0)$. Since $G_{t} \cap \partial D_{\rho}$ is a union of two circular arcs, which both are symmetric with respect to the $x_{2}$-axis, we have

$$
G_{t_{0}} \cap \partial D_{\rho}=\partial D_{\rho}
$$

By the convexity of $G_{t_{0}}$ this implies $D_{\rho} \subset G_{t_{0}}$. But since the flow (4.8) preserves the area we have $\left|G_{t_{0}}\right|=\left|D_{\rho}\right|$. Then it holds $G_{t_{0}}=D_{\rho}$, which is impossible. Therefore the flow $G_{t}$ does not exit the strip $\left\{-\rho<x_{1}<\rho\right\},(4.9)$ holds for all times and the conclusion of the theorem follows.

\section{ACKNLOWDGMENTS}

The research of N.F. has been funded by PRIN Project 2015PA5MP7. The research of V.J. was supported by the Academy of Finland grant 314227. N.F. and M.M. are members of Gruppo Nazionale per l'Analisi Matematica, la Probabilità e le loro Applicazioni (GNAMPA) of INdAM.

\section{REFERENCES}

[1] L. Ambrosio, N. Fusco, D. Pallara. Functions of bounded variation and free discontinuity problems, in the Oxford Mathematical Monographs. The Clarendon Press Oxford University Press, New York (2000).

[2] F. Almgren, J.E. TaYlor, L. Wang, Curvature-driven flows: a variational approach. SIAM J. Control Optim. 31(2), 387-438 (1993).

[3] G. Bellettini, V. Caselles, A. Chambolle, M. Novaga, The volume preserving crystalline mean curvature flow of convex sets in $\mathbb{R}^{n}$. J. Math. Pures Appl. 92 (2009), 499-527.

[4] G. Bellettini, M. Novaga, Comparison results between minimal barriers and viscosity solutions for geometric evolutions. Ann. Scuola Norm. Sup. Pisa Cl. Sci. (4) 26 (1998), 97-131. MR 1632984

[5] G. Bellettini, M. Paolini, Some results on minimal barriers in the sense of De Giorgi applied to driven motion by mean curvature. Rend. Accad. Naz. Sci. XL Mem. Mat. Appl. (5) 19 (1995), 43-67. Errata, ibid. 26 (2002), 161-165.

[6] T. Bonnesen, Les problèmes des isopérimètres et des isépiphanes. Gautier-Villars, Paris, (1929). 
[7] K.A. Brakke, The Motion of a Surface by its Mean Curvature. Math. Notes 20, Princeton Univ. Press, Princeton, NJ (1978).

[8] A. Chambolle, M. Morini, M. Ponsiglione, Nonlocal curvature flows. Arch. Ration. Mech. Anal. 12 (2015), 1263-1329.

[9] A. Chambolle, M. Novaga, Implicit time discretization of the mean curvature flow with a discontinuous forcing term. Interfaces Free Bound. 10 (2008), 283-300.

[10] Y.G. Chen, Y. Giga, S. Goto, Uniqueness and existence of viscosity solutions of generalized mean curvature. Proc. Japan Acad. Ser. A Math. Sci. 65 (1989), 207-210.

[11] M. Cicalese, G.P. LeOnardi, Best constants for the isoperimetric inequality in quantitative form. J. Eur. Math. Soc. 15 (2013), 1101-1129.

[12] E. De Giongi, New ideas in calculus of variations and geometric measure theory. Motion by Mean Curvature and Related Topics (Trento, 1992), de Gruyter, Berlin (1994), 63-69.

[13] M. Delgadino, F. Maggi, Alexandrov's theorem revisited. Anal. PDE 12 (2019), 1613-1642.

[14] N. Dirr, S. Luckhaus, M. Novaga. A stochastic selection principle in case of fattening for curvature flow. Calc. Var. Partial Differential Equations 4 (2001), 405-425.

[15] L.C. Evans, J. Spruck, Motion of level sets by mean curvature I. J. Differential Geom. 33 (1991), 635-681.

[16] Y. Giga, H. Mitake, H.V. Tran, Remarks on large time behavior of level-set mean curvature flow equations with driving and source terms. Discrete Contin. Dyn. Syst. Ser. B (2019), doi: 10.3934/dcdsb.2019228.

[17] Y. Giga, H.V. Tran, L.J. Zhang, On obstacle problem for mean curvature flow with driving force. Geom. Flows 4 (2019), 9-29.

[18] G. Huisken, The volume preserving mean curvature flow. J. Reine Angew. Math. 382 (1987), 35-48.

[19] K. Kasai, Y. Tonegawa, A general regularity theory for weak mean curvature flow. Calc. Var. Partial Differential Equations 50 (2014), 1-68.

[20] I. Kim, D. Kwon, On mean curvature flow with forcing. Comm. Partial Differential Equations 45 (2020), 414-455.

[21] S. Luckhaus, T. Stürzenhecker, Implicit time discretization for the mean curvature flow equation. Calc. Var. Partial Differential Equations 3 (1995), 253-271.

[22] F. MAGGi, Sets of finite perimeter and geometric variational problems. An introduction to geometric measure theory. Cambridge Studies in Advanced Mathematics, 135. Cambridge University Press, Cambridge (2012).

[23] M. Morini, M. Ponsiglione, E. Spadaro Long time behaviour of discrete volume preserving mean curvature flows. Preprint 2020. https://arxiv.org/abs/2004.04799

[24] L. Mugnai, C. Seis, E. Spadaro, Global solutions to the volume-preserving mean-curvature flow. Calc. Var. Partial Differential Equations 55 (2016), Art. 18, 23 pp.

Dipartimento di Matematica e Applicazioni, Università di Napoli "Federico iI", Napoli, Italy E-mail address: n.fusco@unina.it

JyväSkylän Yliopisto, Matematikan Ja Tilastotieteen Laitos, JyväSkylä, Finland.

E-mail address: vesa.julin@jyu.fi

Università di Parma, Dipartimento di Scienze Matematiche, Fisiche e Informatiche, Parma, ITALY

E-mail address: massimiliano.morini@unipr.it 